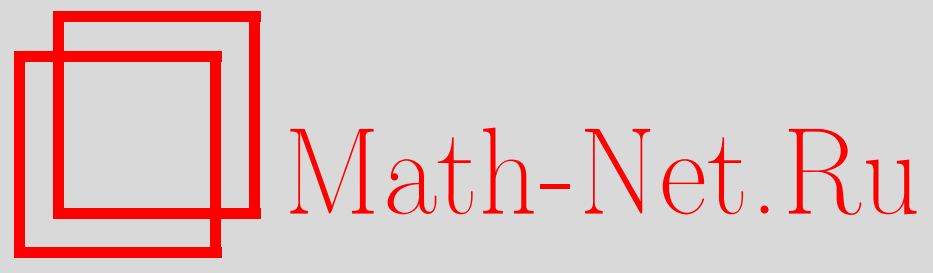

Т. Ф. Киреев, Г. Т. Булгакова, Моделирование напряженного состояния перфорированного цементного кольца, примыкающего к скважине с трещиной гидроразыва пласта, Вестн. Сам. гос. техн. ун-та. Сер. Физ.-мат. науки, 2019, номер 4, 777788

DOI: https://doi.org/10.14498/vsgtu1744

Использование Общероссийского математического портала MathNet.Ru подразумевает, что вы прочитали и согласны с пользовательским соглашением

http: //www . mathnet.ru/rus/agreement

Параметры загрузки:

IP : 54.172 .240 .79

26 апреля 2023 г., 06:31:01

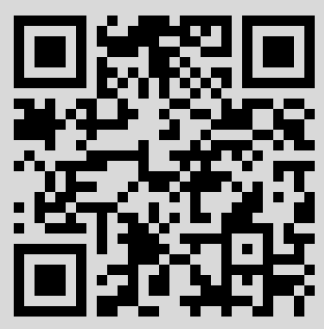


УДК 532.546:519.6

\title{
Моделирование напряженного состояния перфорированного цементного кольца, примыкающего к скважине с трещиной гидроразыва пласта
}

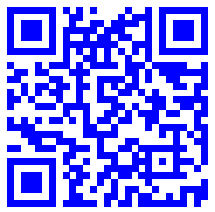

\author{
T. Ф. Киреев ${ }^{1,2}$, Г. Т. Булгакова ${ }^{1}$ \\ 1 Уфимский государственный авиационный технический университет, \\ Россия, 450077, Уфа, ул. К. Маркса, 12. \\ 2 Уфимский научно-технический центр, \\ Россия, 450076, Уфа, ул. Аксакова, 59.
}

\begin{abstract}
Аннотация
Проведено моделирование напряженного состояния перфорированного цементного кольца, примыкающего к скважине с трещиной гидроразрыва пласта. Для вычисления порового давления жидкости используется модель фильтрации несжимаемого флюида. Для вычисления напряженного состояния цементного кольца используется модель линейно-упругого тела и метод конечных объемов с многоточечной аппроксимацией напряжений. Проведена верификация численной модели с помощью сопоставления модельного расчета с расчетом в открытом пакете моделирования Fenics. Показано, что максимальное значение напряжения Мизеса приходится на зону перфораций на стыке цементного кольца и эксплуатационной колонны, а наличие трещины гидроразрыва пласта может снижать напряжение цементного кольца.
\end{abstract}

Ключевые слова: напряженное состояние, цементное кольцо, заколонные перетоки, трещина гидроразрыва пласта, многоточечная аппроксимация напряжений, сетка Вороного.

Получение: 13 сентября 2019 г. / Исправление: 28 октября 2019 г. / Принятие: 11 ноября 2019 г. / Публикация онлайн: 23 декабря 2019 г.

\section{Краткое сообщение}

ə () (i) Контент публикуется на условиях лицензии Creative Commons Attribution 4.0 International (https://creativecommons.org/licenses/by/4.0/deed.ru)

\section{Образец для цитирования}

Kireev T. F., Bulgakova G. T. Modeling of stress state of a perforated cement sheath in a well with hydraulic fracture, Vestn. Samar. Gos. Tekhn. Univ., Ser. Fiz.-Mat. Nauki [J. Samara State Tech. Univ., Ser. Phys. Math. Sci.], 2019, vol. 23, no. 4, pp. 777-788. doi: 10.14498 /vsgtu1744.

\section{Сведения об авторах}

Тимур Фаритович Киреев (1) https://orcid.org/0000-0002-2653-1059

аспирант; каф. математики ; ведущий инженер-аналитик; отд. математического моделирования ${ }^{2}$; e-mail:kireevtf@mail.ru

Гузель Талгатовна Булгакова (D) https://orcid.org/0000-0001-8030-1791

доктор физико-математических наук, профессор; каф. математики;

e-mail: bulgakova.guzel@mail.ru 
Введение. Одной из насущных проблем в нефтегазовой отрасли является разрушение цементного кольца между породой и эксплуатационной колонной скважины. Это приводит к преждевременному обводнению скважин и загрязнению окружающей среды. В данной работе проводится моделирование прочности цементного кольца с помощью вычисления напряжений Мизеса в каждой точке цементного кольца и эксплуатационной колонны. Области пласта с максимальным напряжением Мизеса являются наименее прочными.

Моделирование прочности цементного кольца проводится с помощью неполно связных задач линейной упругости и фильтрации Дарси. Используются следующие предположения:

1) порода пласта является недеформируемой;

2) трещина гидроразрыва пласта (ГРП) примыкает к цементному кольцу, является бесконечнопроводимой и недеформируемой;

3) цементное кольцо и эксплуатационная колонна деформируемы и представляют собой линейно-упругое тело;

4) между цементным кольцом и эксплуатационной колонной выполняются условия идеального контакта;

5) забойное давление внутри скважины действует на внутреннюю стенку эксплуатационной колонны;

6) давление со стороны пласта на внешнюю стенку цементного кольца определяется только поровым давлением жидкости в пласте;

7) деформации цементного кольца и эксплуатационной колонны малы и не оказывают влияния на характер движения флюида в пласте и в скважине.

Алгоритм решения задачи состоит из двух последовательных шагов:

1) вычисление стационарного распределения пластового давления с помощью решения уравнения фильтрации несжимаемого флюида;

2) вычисление напряженного состояния цементного кольца и эксплуатационной колонны с помощью решения стационарного уравнения линейной упругости, в котором в качестве граничного условия задано пластовое давление, вычисленное в пункте 1.

Пространственная дискретизация уравнений осуществляется с помощью построения трехмерной расчетной сетки Вороного [1] для пласта, трещины ГРП, цементного кольца и эксплуатационной колонны. Для решения уравнения фильтрации применяется метод конечных объемов с двухточечной аппроксимацией потока, а для решения уравнения упругости - метод конечных объемов с многоточечной аппроксимацией напряжений [2,3].

1. Задача фильтрации. Задача фильтрации описывается следующим образом:

$$
\begin{gathered}
\operatorname{div}\left(\frac{k}{\mu} \operatorname{grad}(p)\right)=0 \\
\left.p\right|_{\text {perf }}=p_{\text {well }},\left.\quad p\right|_{\text {frac }}=p_{\text {well }},\left.\quad \frac{\partial p}{\partial n}\right|_{\text {boundary }}=0 .
\end{gathered}
$$

Здесь $p$ - пластовое давление, $k$ - проницаемость пласта, $\mu-$ вязкость флюида, $p_{\text {well }}$ - забойное давление скважины, perf - перфорации скважины, frac трещина ГРП, boundary - граница пласта, которая включает в себя внутреннюю часть, внешнюю часть, а также кровлю и подошву пласта. Внешняя 
часть границы пласта описывает границу области дренирования, а внутренняя часть границы пласта представляет собой внешнюю стенку цементного кольца скважины.

2. Задача упругости. Цементное кольцо и эксплуатационная колонна представляют собой единый полый цилиндр, как показано на рис. 1.

Напряженное состояние цементного кольца и эксплуатационной колонны описывается так:

$$
\begin{gathered}
\nabla \cdot \boldsymbol{\sigma}+\vec{f}=\overrightarrow{0}, \quad \boldsymbol{\sigma}=\mathbf{C} \boldsymbol{\varepsilon}, \quad \boldsymbol{\varepsilon}=\frac{1}{2}\left(\nabla \vec{u}+(\nabla \vec{u})^{\top}\right), \\
\left.\vec{f}\right|_{S_{1}}=-p \vec{n},\left.\quad \vec{f}\right|_{S_{2}}=-p_{\text {well }} \vec{n},\left.\quad \vec{f}\right|_{S_{3}}=-p_{\text {well }} \vec{n},\left.\quad \vec{u}\right|_{S_{4}}=\overrightarrow{0} .
\end{gathered}
$$

Здесь $\boldsymbol{\sigma}$ - симметричный тензор напряжений (2 ранга); $\vec{f}$-внешние силы; $\mathbf{C}$ - тензор жесткости (4 ранга); $\boldsymbol{\varepsilon}$ - симметричный тензор деформации (2 ранга); $\vec{u}=\left(u_{x}, u_{y}, u_{z}\right)^{\top}$ - вектор перемещений; $u_{x}, u_{y}, u_{z}$ - перемещения вдоль осей $x, y, z$ соответственно; $S_{1}$ - внешняя стенка цементного кольца (рис. 1$)$; $S_{2}$ - внутренняя стенка эксплуатационной колонны; $S_{3}$ - стенки перфораций в цементном кольце и эксплуатационной колонне; $S_{4}$ - торцы цементного кольца и эксплуатационной колонны; $\vec{n}$ - поле внешних нормалей к цементному кольцу и эксплуатационной колонне.

Продемонстрируем решение задачи упругости. Проинтегрируем уравнение упругости в ячейке $i$ :

$$
\begin{gathered}
\int_{V_{i}} \nabla \cdot \boldsymbol{\sigma} d V+\int_{V_{i}} \vec{f} d V=\overrightarrow{0}, \\
\int_{\partial V_{i}} \boldsymbol{\sigma} \cdot \vec{n} d S+\overrightarrow{f_{i}}=\overrightarrow{0}, \\
\sum_{j \in \Psi(i)} \int_{S_{i j}} \boldsymbol{\sigma} \cdot \vec{n} d S+\overrightarrow{f_{i}}=\overrightarrow{0} .
\end{gathered}
$$

Здесь $V_{i}$ - объем ячейки $i ; f_{i}$ - среднее значение внешней силы, действующей на ячейку $i$. Интеграл по поверхности $\partial V_{i}$ ячейки $i$ представляется в виде

Рис. 1. Цементное кольцо и эксплуатационная колонна. Голубым цветом закрашена внешняя стенка цементного кольца, зеленым - внутренняя стенка эксплуатационной колонны, красным - стенки перфораций, серым - торцы цементного кольца и эксплуатационной колонны, желтым - трещина ГРП (онлайн в цвете)

[Figure 1 (color online). Cement sheath and production casing. The outer wall of the cement sheath is blue, the inner wall of the production casing is green, the perforation walls are red, the ends of the cement sheath and the production casing are gray, and the hydraulic fracture is yellow]

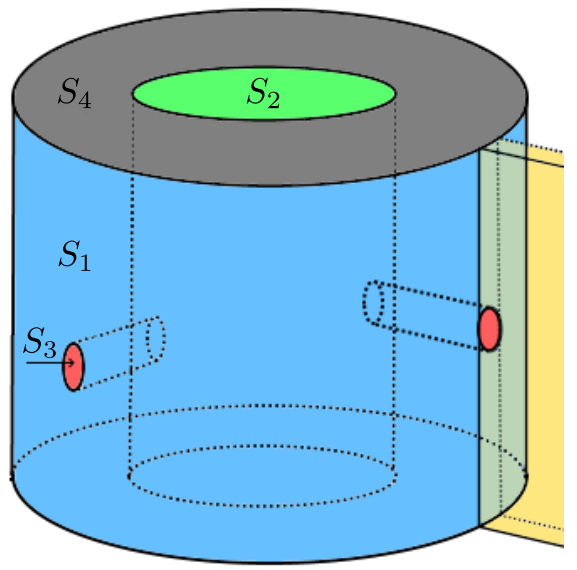


суммы поверхностных интегралов по каждой грани этой ячейки. Множество ячеек, смежных с $i$-той ячейкой, обозначается $\Psi(i) ; S_{i j}$ - грань между $i$-той и $j$-той ячейками.

Пусть $(\boldsymbol{\sigma} \cdot \vec{n})_{i j}$ - среднее нормальное напряжение на грани между $i$-той и $j$-той ячейками. Тогда последнее уравнение будет иметь вид

$$
\sum_{j \in \Psi(i)}\left|S_{i j}\right|(\boldsymbol{\sigma} \cdot \vec{n})_{i j}+\overrightarrow{f_{i}}=\overrightarrow{0}
$$

Введем также среднее значение $\vec{u}_{i}$ перемещения в ячейке $i$. Метод многоточечной аппроксимации напряжений заключается в том, чтобы искать значение $\left|S_{i j}\right|(\boldsymbol{\sigma} \cdot \vec{n})_{i j}$ на грани между $i$-той и $j$-той ячейками в виде линейной комбинации некоторого набора $\vec{u}_{k}$ :

$$
\left|S_{i j}\right|(\boldsymbol{\sigma} \cdot \vec{n})_{i j}=\sum_{v \in V(i, j)} \sum_{k \in C(v)} \boldsymbol{t}_{i j k v} \vec{u}_{k} .
$$

Здесь $\boldsymbol{t}_{i j k v}$ - тензоры 2-го ранга; $V(i, j)$ - множество всех вершин грани $i j$; $C(v)$ - множество всех ячеек, смежных с вершиной $v ;\left|S_{i j}\right|$ - площадь грани $S_{i j}$.

Каждой вершине $v$ расчетной сетки соответствует одна ячейка дуальной сетки. Для каждой дуальной ячейки решается специальная локальная задача для определения коэффициентов $\boldsymbol{t}_{i j k v}$ внутри этой дуальной ячейки. Когда все коэффициенты $\boldsymbol{t}_{i j k v}$ будут вычислены, уравнения равновесия во всех ячейках можно будет представить в виде системы линейных уравнений относительно неизвестных $\vec{u}_{i}$.

3. Решение локальной задачи упругости. Для каждой вершины сетки решается отдельная локальная задача. Рассмотрим вершину $v$ и ячейку $i$, инцидентную этой вершине. Рассмотрим все ребра и все грани сетки, которые инциденты одновременно вершине $v$ и ячейке $i$. Многогранник, натянутый на вершину $v$, центроид ячейки $i$ и центроиды всех этих ребер и граней, называется мини-ячейкой и обозначается $(i, v)$ (рис. 2$)$. Грань мини-ячейки $(i, v)$, инцидентная какой-либо другой ячейке $j$, называется мини-гранью и обозначается $(i, j, v)$. Очевидно, что все ячейки сетки можно представить в виде объединения некоторого множества мини-ячеек, а все грани - в виде объединения некоторого множества мини-граней.

Пусть в каждой точке $\vec{r}$ мини-ячейки $(i, v)$ каждая компонента вектора перемещений $\vec{u}(\vec{r})=\left(u_{x}(\vec{r}), u_{y}(\vec{r}), u_{z}(\vec{r})\right)^{\top}$ является линейной функцией координат:

$$
\begin{aligned}
& u_{x}(\vec{r})=u_{i, x}+\nabla u_{i v}, x \cdot\left(\vec{r}-\vec{r}_{i}\right), \\
& u_{y}(\vec{r})=u_{i, y}+\nabla u_{i v}, y \cdot\left(\vec{r}-\vec{r}_{i}\right), \\
& u_{z}(\vec{r})=u_{i, z}+\nabla u_{i v}, z \cdot\left(\vec{r}-\vec{r}_{i}\right) .
\end{aligned}
$$

Здесь $\vec{u}_{i}=\left(u_{i, x}, u_{i, y}, u_{i}, z\right)^{\top}$ - среднее значение перемещения в $i$-той ячейке; $\nabla u_{i v}, x, \nabla u_{i v}, y, \nabla u_{i v}, z$ - градиенты компонент вектора перемещений (это векторы), $\vec{r}_{i}$ - центр ячейки $i$. Считаем, что градиенты $\nabla u_{i v, x}, \nabla u_{i v, y}, \nabla u_{i v, z}$ в данной мини-ячейке - константы, т.е. они не зависят от координаты $\vec{r}$. Поэтому тензор напряжений $\sigma_{i v}$ в мини-ячейке $(i, v)$ тоже не зависит от $\vec{r}$. 
Рис. 2. Несколько ячеек, инцидентных вершине $v$. Ребра ячеек изображены черными отрезками.

Мини-ячейка $(i, v)$ закрашена серым цветом

[Figure 2. Cells incident to the vertex $v$. Cell edges are depicted by black segments. The subcell $(i, v)$ is shaded gray]

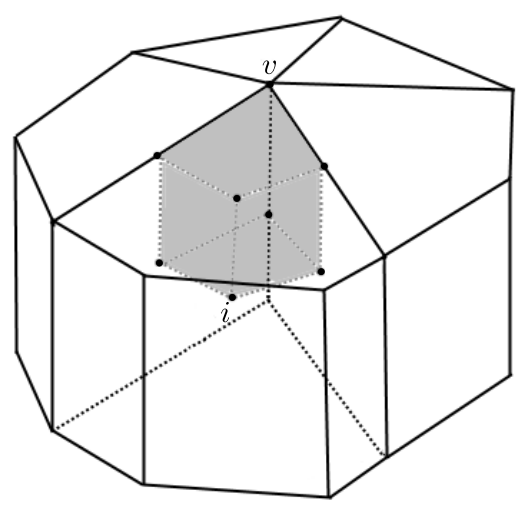

Пусть вершина $v$ инцидентна $N$ ячейкам и $M$ граням. На каждой миниграни $m$ (меняется от 1 до $M$ ) задаем условие непрерывности напряжения (на всей мини-грани) и условие непрерывности перемещения (в единственной точке $\vec{r}_{m}$ мини-грани):

$$
\begin{array}{ll}
(\boldsymbol{\sigma} \cdot \vec{n})_{i j v}=-(\boldsymbol{\sigma} \cdot \vec{n})_{j i v}, & (i, j) \in \operatorname{Neighbours}(m), \\
\vec{u}_{i}+\nabla \vec{u}_{i v} \cdot\left(\vec{r}_{m}-\vec{r}_{i}\right)=\vec{u}_{j}+\nabla \vec{u}_{j v} \cdot\left(\vec{r}_{m}-\vec{r}_{j}\right), & (i, j) \in \operatorname{Neighbours}(m) .
\end{array}
$$

Это $2 M$ векторных уравнений, то есть $6 M$ скалярных уравнений. Неизвестные переменные - это градиенты $\nabla \vec{u}_{i v}$ в каждой мини-ячейке. В данной локальной задаче имеем $N$ мини-ячеек - это $N$ неизвестных градиентов, или $9 N$ неизвестных скаляров. Эта система уравнений имеет единственное решение, если $2 M=3 N$. Для простых трехмерных сеток, состоящих из параллелепипедов, призм или тетраэдров, это условие всегда выполняется. Но для сеток, состоящих из многогранников произвольной формы (в частности, для сетки Вороного в общем случае) это условие не выполняется.

Пусть $\boldsymbol{\sigma}_{i v}=\left\{\sigma_{i v}, p q\right\}, \mathbf{C}_{i v}=\left\{c_{i v}, p q r s\right\}, \boldsymbol{\varepsilon}_{i v}=\left\{\varepsilon_{i v}, r s\right\}$, где $p, q, r, s$-индексы компонент тензоров, принимающие значения от 1 до 3 . Тогда

$$
\sigma_{i v, p q}=\sum_{r=1}^{3} \sum_{s=1}^{3} c_{i v}, p q r s \varepsilon_{i v}, r s .
$$

Представим эту систему уравнений в виде

$$
\left(\begin{array}{cc}
\mathbf{C}_{i v} \cdot \vec{n}_{i j v} & 0 \\
\left(\vec{r}_{m}-\vec{r}_{i}\right) & \pm 1 \\
0 & I
\end{array}\right)\left(\begin{array}{c}
\nabla \vec{u}_{i v} \\
\vec{u}_{i}
\end{array}\right)=\left(\begin{array}{l}
0 \\
0 \\
1
\end{array}\right)
$$

или

$$
\mathbf{A} \vec{x}=\vec{b}
$$

Вычислив псевдообратную матрицу $\mathbf{A}^{-1}$, мы сможем представить неизвестные градиенты $\nabla \vec{u}_{i v}$ в виде линейных комбинаций переменных $\vec{u}_{i}$ :

$$
\nabla u_{i v, r s}=\sum_{k=1}^{N} \vec{\alpha}_{i v, r s, k} \cdot \vec{u}_{k},
$$


где $\vec{\alpha}_{i v}, r s, k-$ вектор длины 3 (является блоком матрицы $\mathbf{A}^{-1}$ ). Таким образом, для произвольных трехмерных сеток градиенты $\nabla \vec{u}_{i v}$ выражаются через $\vec{u}_{i}$ в смысле минимизации невязки $\|\mathbf{A} \vec{x}-\vec{b}\|$. Для простых трехмерных сеток, состоящих из параллелепипедов, призм или тетраэдров, $\nabla \vec{u}_{i v}$ выражаются через $\vec{u}_{i}$ точно.

Подставляем полученные $\nabla \vec{u}_{i v}$ в выражение для напряжения на миниграни, чтобы выразить это напряжение через неизвестные переменные $\vec{u}_{k}$ :

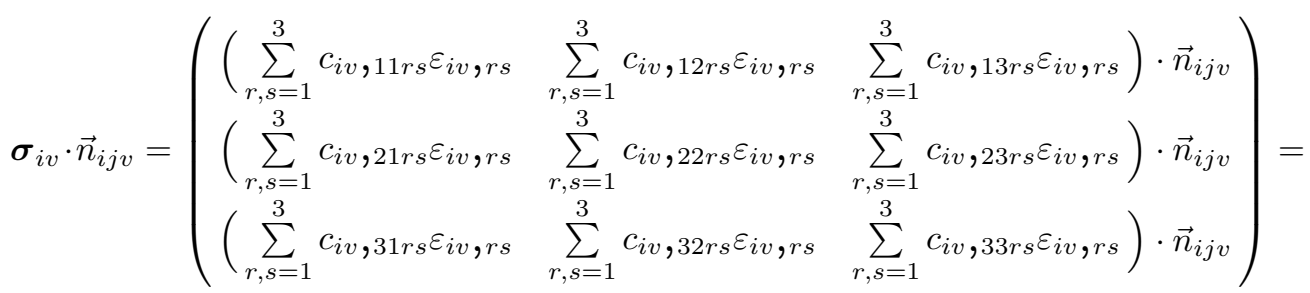

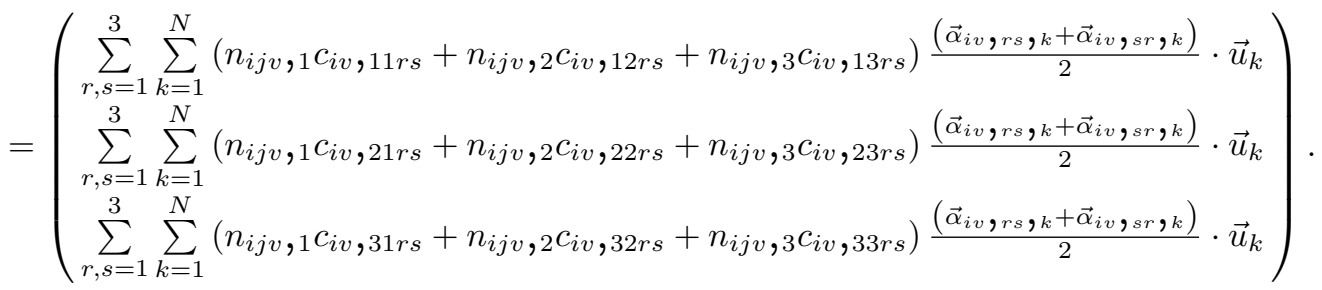

Если в последнем выражении каждый вектор $\vec{\alpha}_{i v}, r s, k$ представить в виде строки, то получим представление напряжения на мини-грани в виде линейной комбинации $\vec{u}_{k}$ :

$$
\boldsymbol{\sigma}_{i v} \cdot \vec{n}_{i j v}=\sum_{k=1}^{N} \boldsymbol{t}_{i j k v} \vec{u}_{k}
$$

Напряжение на всей грани $i j$ определяется так:

$$
\boldsymbol{\sigma}_{i j} \cdot \vec{n}_{i j}=\sum_{v \in V(i, j)} \boldsymbol{\sigma}_{i v} \cdot \vec{n}_{i j v}=\sum_{v \in V(i, j)} \sum_{k \in C(v)} \boldsymbol{t}_{i j k v} \vec{u}_{k} .
$$

В данной работе для решения локальных задач на границе сетки используется условие нулевого напряжения на границе. Для этого вокруг границы сетки создается слой вспомогательных ячеек с нулевым тензором жесткости, и все локальные задачи решаются описанным выше способом для внутренних вершин сетки.

4. Решение глобальной задачи упругости. Для каждой ячейки получаем линейное уравнение относительно неизвестных перемещений $\vec{u}_{i}$ в каждой ячейке:

$$
\sum_{j \in \Psi(i)} \sum_{v \in V(i, j)} \sum_{k \in C(v)} \boldsymbol{t}_{i j k v} \vec{u}_{k}+\vec{f}_{i}=\overrightarrow{0}
$$

Поскольку градиент

$$
\nabla u_{i v, r s}=\sum_{k=1}^{N} \vec{\alpha}_{i v}, r s, k \cdot \vec{u}_{k}
$$


в каждой мини-ячейке известен, можно вычислить тензор напряжений $\boldsymbol{\sigma}_{i v}$ и напряжение Мизеса $\sigma_{m}, i v$ в каждой мини-ячейке:

$$
\begin{aligned}
& \mathbf{S}_{i v}=\boldsymbol{\sigma}_{i v}-\frac{1}{3} \operatorname{trace}\left(\boldsymbol{\sigma}_{i v}\right) \mathbf{I},
\end{aligned}
$$

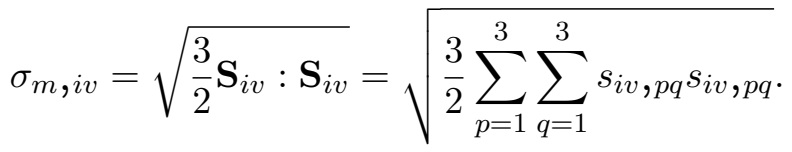

Напряжение Мизеса в ячейке $i$ принимается равным среднему значению напряжения Мизеса по всем мини-ячейкам $(i, v)$.

5. Вычислительный эксперимент. Рассмотрены добывающая и нагнетательная вертикальные скважины с трещинами ГРП, находящиеся друг от друга на расстоянии 500 м и работающие с постоянными забойными давлениями 50 и 150 атм соответственно. Вычисляется напряженное состояние цементного кольца и эксплуатационной колонны для добывающей скважины. Толщина пласта -3 м, внутренний радиус эксплуатационной колонны - 6 см, толщина эксплуатационной колонны - 1.8 см, толщина цементного кольца 3 см, полудлина трещин ГРП - 125 м, ширина раскрытия трещин ГРП1 см, проницаемость пласта - 100 миллидарси, вязкость флюида - 1, коэффициент Пуассона цементного кольца и эксплуатационной колонны - 0.25 , модуль Юнга цементного кольца - 5 ГПа, модуль Юнга эксплуатационной колонны - 200 ГПа.

Расчетная сетка состоит из ячеек пласта, ячеек трещины ГРП, ячеек цементного кольца и ячеек эксплуатационной колонны для обеих скважин (рис. 3). Перфорации представляют собой пустоты в цементном кольце и эксплуатационной колонне, имеющие форму параллелепипедов со сторонами 5 см. Количество пластовых ячеек - 200 тысяч, количество ячеек цеметного кольца и эксплуатационной колоны (для одной скважины) - 10 тысяч.

На рис. 4 показано распределение пластового давления около добывающей скважины при наличии и при отсутствии трещины ГРП.

На рис. 5 видно, что смещения цементного кольца и эксплуатационной колонны в горизонтальной плоскости примерно одинаковы по всей высоте пласта (при наличии и при отсутствии трещины ГРП).

На рис. 6 и 7 показано напряжение Мизеса в цементном кольце и эксплуатационной колонне. Видно, что самое большое напряжение Мизеса приходится на зону перфораций на стыке цементного кольца и эксплуатационной

Рис. 3. Расчетная сетка с локальным измельчением вблизи двух скважин; слева - нагнетательная скважина, справа добывающая скважина

[Figure 3. Voronoi grid with refinement near two wells; left - the injection well, right - the production well]

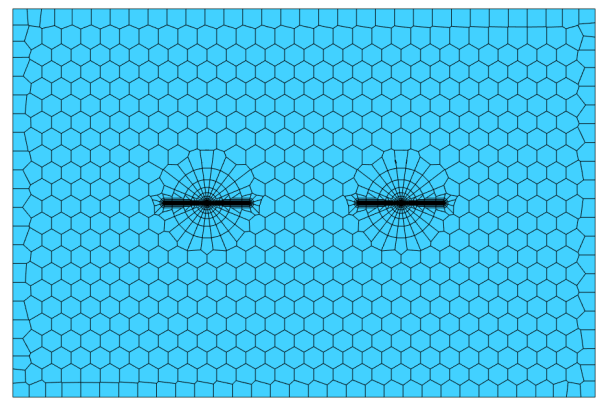




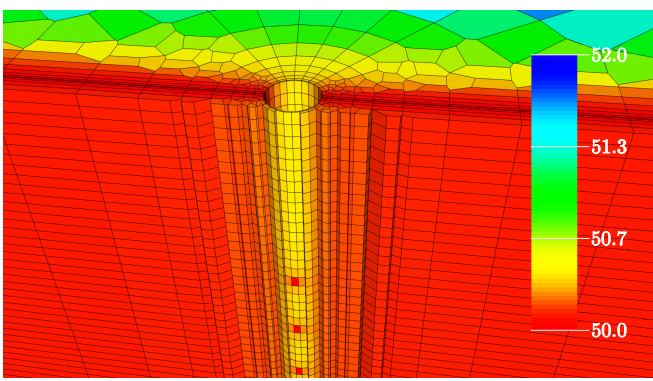

a

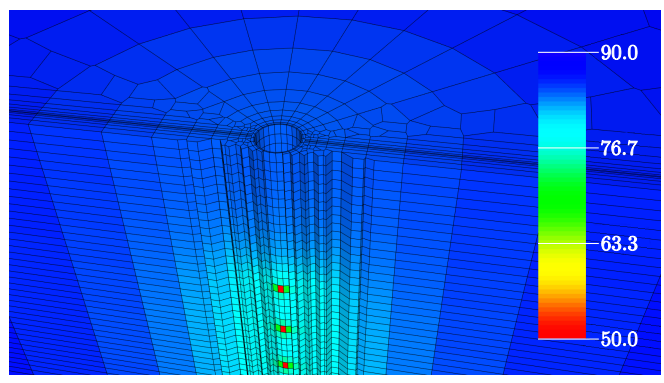

$b$

Рис. 4. Распределение пластового давления (атм) вблизи добывающей скважины при наличии (a) и при отсутствии $(b)$ трещины ГРП (онлайн в цвете)

[Figure 4 (color online). The distribution of reservoir pressure (atm) near the production well with (a) and without (b) hydraulic fracture]

Рис. 5. Перемещение точек цементного кольца и эксплуатационной колонны вдоль горизонтальной оси (мкм) (онлайн в цвете)

[Figure 5 (color online). Displacements $(\mu \mathrm{m})$ of the cement sheath and production casing along the horizontal axis]

Рис. 6. Напряжение Мизеса (атм) в каждой точке цементного кольца и эксплуатационной колонны (при наличии трещины ГРП) (онлайн в цвете)

[Figure 6 (color online). Von Mises stress (atm) at each point of the cement sheath and production casing (in the presence of hydraulic fracture)]

Рис. 7. Напряжение Мизеса (атм) в каждой точке цементного кольца и эксплуатационной колонны (при отсутствии трещины ГРП) (онлайн в цвете)

[Figure 7 (color online). Von Mises stress (atm) at each point of the cement sheath and production casing (in the absence of hydraulic fracture)]
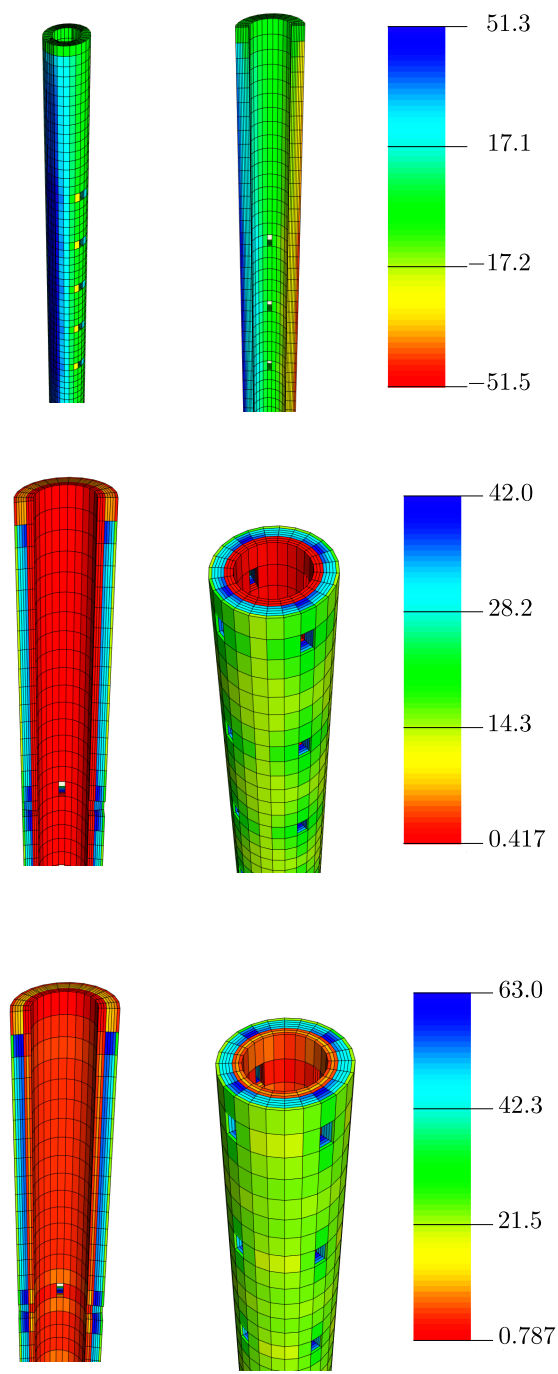
колонны (синие области). Если напряжение Мизеса превысит предел прочности цемента, то цементное кольцо начнет разрушаться в этой зоне. Вдоль внутренней стенки цементного кольца от перфораций могут пойти трещины, которые в итоге станут причиной заколонных перетоков.

На торцевой части цилиндра также наблюдается высокое значение напряжения Мизеса, поскольку торцы закреплены и неподвижны. Напряжение в цементном кольце существенно выше напряжения в эксплуатационной колонне (это связано с тем, что цементное кольцо имеет меньший модуль Юнга).

Сравнивая рис. 6 и 7, можно заметить, что наличие трещины ГРП не меняет картину пространственного распределения напряжений в цементом кольце и эксплуатационной колонне, но уменьшает максимальное значение напряжения в 1.5 раза.

6. Верификация численного решения уравнения упругости. Для верификации численного метода рассмотрена задача деформации балки под собственной тяжестью. Балка состоит из изотропного материала и закреплена на одном конце. На каждую точку балки действует сила тяжести.

Проведено сравнение расчета, полученного с помощью разработанного численного метода, с расчетом в открытом пакете моделирования Fenics [4], в котором используется метод конечных элементов. В первом расчете использовалась структурированная сетка (hex mesh), состоящая из 3840 ячеек кубической формы, а во втором расчете - тетраэдральная сетка, состоящая из $3840 \times 6$ элементов.

На рис. 8 показаны деформированное состояние балки и напряжения Мизеса в каждой точке балки, полученные в двух расчетах. Видно, что деформация и напряжения Мизеса близки друг к другу в двух расчетах в каждой точке балки.

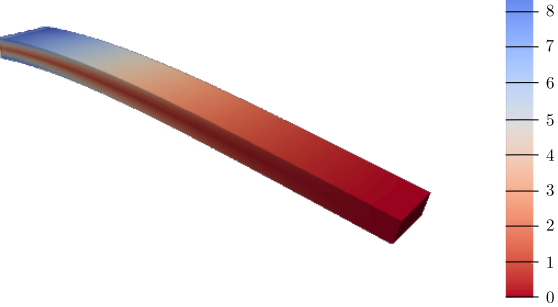

a
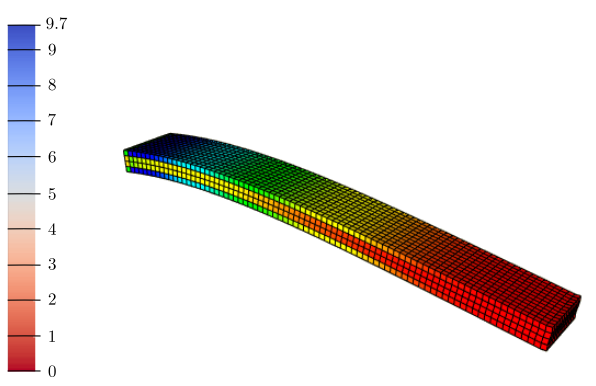

$b$

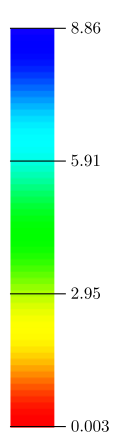

Рис. 8. Напряжение Мизеса в каждой точке деформированной балки: (а) расчет в пакете моделирования Fenics, (b) наш расчет

[Figure 8. Von Mises stress at each point of the deformed beam: (a) calculation in the Fenics package, (b) our calculation]

Заключение. Проведено моделирование напряженного состояния перфорированного цементного кольца, примыкающего к добывающей скважине. Показано, что максимальное значение напряжения Мизеса приходится на зону перфораций на стыке цементного кольца и эксплуатационной колонны, а наличие трещины гидроразрыва пласта может снижать напряжение цементного кольца. 
Конкурирующие интересы. Мы не имеем конкурирующих интересов.

Авторский вклад и ответственность. Все авторы принимали участие в разработке концепции статьи и в написании рукописи. Авторы несут полную ответственность за предоставление окончательной рукописи в печать. Окончательная версия рукописи была одобрена всеми авторами.

Финансирование. Работа выполнена при поддержке ООО «Уфимский НТЦ» и РФФИ (проект № 17-41-020226-р_а).

\section{Библиографический список}

1. Palagi C. L., Aziz K. Use of Voronoi grid in reservoir simulation // SPE Advanced Technology Series, 1994. vol. 20, no. 2. pp. 69-77. doi: 10.2118/22889-PA.

2. Nordbotten J. M. Cell-centered finite volume discretizations for deformable porous media // Int. J. Numer. Methods Eng., 2014. vol. 100, no. 6. pp. 399-418. doi: 10.1002/nme. 4734.

3. Keilegavlen E., Nordbotten J. M. Finite volume methods for elasticity with weak symmetry// Int. J. Numer. Methods Eng., 2017. vol.112, no.8. pp. 939-962. doi:10.1002/nme. 5538.

4. Alnæs M. et al. The FEniCS Project Version 1.5// Archive of Numerical Software, 2015. vol. 3, no. 100. doi: 10.11588/ans.2015.100.20553. 
MSC: 76S05, 74A10

\title{
Modeling of stress state of a perforated cement sheath in a well with hydraulic fracture
}

\author{
T. F. Kireev ${ }^{1,2}$, G. T. Bulgakova ${ }^{1}$ \\ 1 Ufa State Aviation Technical University, \\ 12, K. Marx st., Ufa, 450077, Russian Federation. \\ 2 Ufa Scientific and Technical Center, \\ 59, Aksakov st., Ufa, 450076, Russian Federation.
}

\begin{abstract}
Modeling of stress state of a perforated cement sheath in a well with hydraulic fracture is performed. The incompressible fluid flow model is used to calculate the pore pressure of a fluid. The linear-elastic body model and finite volume method with multipoint stress approximation are used to calculate the stress state of the cement sheath and production casing. The numerical model was verified by comparing the calculation results with a calculation in the Fenics open-source computing platform. It is shown that the maximum value of von Mises stress falls on the perforation zone at the junction of the cement sheath and the production casing. The presence of a hydraulic fracture can reduce the stress of the cement sheath.
\end{abstract}

Keywords: stress state, cement sheath, behind-the-casing flows, hydraulic fracture, multipoint stress approximation, Voronoi grid.

Received: $13^{\text {th }}$ September, 2019 / Revised: $28^{\text {th }}$ October, $2019 /$ Accepted: $11^{\text {th }}$ November, 2019 / First online: $23^{\text {rd }}$ December, 2019

Competing interests. We have no competing interests.

Authors' contributions and responsibilities. Each author has participated in the article concept development and in the manuscript writing. The authors are absolutely responsible for submitting the final manuscript in print. Each author has approved the final version of manuscript.

Funding. This research was supported by UNTC LLC and RFBR (project no. 17-41020226-r_a).

\section{Short Communication}

ㅇ (ㄱ) The content is published under the terms of the Creative Commons Attribution 4.0 International License (http://creativecommons.org/licenses/by/4.0/)

Please cite this article in press as:

Kireev T. F., Bulgakova G. T. Modeling of stress state of a perforated cement sheath in a well with hydraulic fracture, Vestn. Samar. Gos. Tekhn. Univ., Ser. Fiz.-Mat. Nauki [J. Samara State Tech. Univ., Ser. Phys. Math. Sci.], 2019, vol. 23, no. 4, pp. 777-788. doi: $10.14498 /$ vsgtu1744.

\section{Authors' Details:}

Timur F. Kireev (1) https://orcid.org/0000-0002-2653-1059

Postgraduate Student; Dept. of Mathematics ${ }^{1}$; Lead Engineer/Analyst; Dep. of Mathematical Modeling $^{2}$; e-mail: kireevtf@mail.ru

Guzel T. Bulgakova (1) https://orcid.org/0000-0001-8030-1791

Dr. Phys. \& Math. Sci., Professor; Dept. of Mathematics; e-mail: bulgakova.guzel@mail.ru 


\section{References}

1. Palagi C. L., Aziz K. Use of Voronoi grid in reservoir simulation, SPE Advanced Technology Series, 1994, vol. 20, no. 2, pp. 69-77. doi: 10.2118/22889-PA.

2. Nordbotten J. M. Cell-centered finite volume discretizations for deformable porous media, Int. J. Numer. Methods Eng., 2014, vol. 100, no. 6, pp. 399-418. doi: 10.1002/nme.4734.

3. Keilegavlen E., Nordbotten J. M. Finite volume methods for elasticity with weak symmetry, Int. J. Numer. Methods Eng., 2017, vol. 112, no. 8, pp. 939-962. doi: 10.1002/nme.5538.

4. Alnæs M. et al. The FEniCS Project Version 1.5, Archive of Numerical Software, 2015, vol. 3, no. 100. doi: 10.11588/ans.2015.100.20553. 